\title{
Composition of working groups: How does the functional and social diversity affect their performance?
}

DOI: $10.46932 / \mathrm{sfjdv} 2 \mathrm{n} 2-100$

Received in: March 1st, 2021

Accepted in: May 30th, 2021

\author{
Leticia Pérez-Calero \\ Department of Management and Marketing \\ Pablo de Olavide University \\ Carretera de Utrera, Km.1, 41013, Seville (Spain) \\ E-mail: lcalero@upo.es \\ Rocio Aguilar \\ Department of Management and Marketing \\ Pablo de Olavide University \\ Carretera de Utrera, Km.1, 41013, Seville (Spain) \\ E-mail: ragucar@upo.es \\ Barbara Larrañeta \\ Department of Management and Marketing \\ Pablo de Olavide University \\ Carretera de Utrera, Km.1, 41013, Seville (Spain) \\ E-mail: blargom@upo.es
}

\section{RESUMEN}

Este trabajo examina cómo la configuración de un equipo de trabajo, en base a las características de sus miembros, puede repercutir en su rendimiento. En concreto, nos centramos en la diversidad como elemento a tener en cuenta en la configuración de los equipos. Mediante información recogida a través de encuestas a los estudiantes, concluimos que los diferentes tipos de diversidad analizados van a tener influencias diferentes en los rendimientos del grupo por lo que las nuevas configuraciones deben tener en cuenta grupos diversos en género, con la misma nacionalidad, con estudios previos similares y con experiencia laboral en el mismo nivel. Estos resultados contribuyen a considerar que los resultados de equipo de trabajo no dependen sólo de la suma de los perfiles de sus individuos, sino de la integración de tales recursos aportados y la voluntad de trabajar de forma efectiva con los demás de manera que contribuya al logro de sus metas.

Palabras claves: grupos diversos, rendimiento, enseñanza universitaria.

\section{INTRODUCCIÓN}

Para poder adaptarnos al Espacio Europeo de Educación Superior (EEES) debemos enseñar a los futuros graduados, además de conocimientos teóricos, un conjunto de destrezas y habilidades que les permitan desarrollar su carrera profesional en un mundo cada vez más globalizado (Arroyo-Salgueira et al., 2021). En relación a este último aspecto, la existencia de las Enseñanzas Practicas y de Desarrollo (EPD) supone un refuerzo a la docencia tradicional, donde ya no sólo es el profesor el que tiene una labor 
encomendada, sino que los alumnos se implican y participan activamente en el desarrollo de las clases a través de la elaboración de casos y trabajos sobre situaciones y empresas reales.

La realización de las EPDs se desarrolla mediante equipos de trabajo, cuyo funcionamiento y composición es un elemento clave a tener en cuenta ya que afecta a su rendimiento. Sin embargo, mientras las características propias de cada asignatura, la formación de los alumnos y las condiciones del entorno físico y académico en que se ejerce la docencia, han sido principales factores a considerar en muchos de los proyectos de innovación docente presentados en esta y otras universidades, las características de los grupos de trabajo y de los perfiles de los miembros que lo integran ha sido casi obviado. Hasta la fecha se han tratado a los grupos de trabajo como entidades homogéneas, sin considerar las características sociales y humanas de cada uno de los alumnos que los integran y que van a repercutir en su rendimiento. Es más, la manera en que se conforman los grupos no ha seguido unos criterios objetivos y ha estado centrada en: 1) la elección libre del alumno, 2) la aleatoriedad del profesor, siendo pocas las veces en que se trata de una decisión deliberada y justificada.

Sin embargo, es importante considerar al equipo de trabajo como grupo cuyos resultados no dependen sólo de la suma de los perfiles de sus individuos, sino de la integración de tales recursos aportados y la voluntad de trabajar de forma efectiva con los demás de manera que contribuya al logro de las metas de ese equipo.

La importancia de la composición de los equipos de trabajo se pone de manifiesto en los crecientes esfuerzos realizados por distintas empresas para explorar el perfil de sus trabajadores, durante los procesos de selección y diseño organizativo.

Todos los aspectos mencionados señalan la necesidad de llevar a cabo una iniciativa desde las aulas que permita conocer las implicaciones de la composición de los grupos de trabajo para su rendimiento.

\section{OBJETIVOS}

El objetivo general de este proyecto es demostrar cómo la configuración de un equipo de trabajo, en base a las características de sus miembros, puede repercutir en su rendimiento. En concreto, nos centramos en la diversidad como elemento a tener en cuenta en la configuración de los equipos.

La diversidad hace referencia a las diferencias entre individuos sobre la base de cualquier atributo que podría dirigir a la percepción de que otra persona es diferencia a uno mismo (Jackson, 1992; Triandis et al. 1994; Williams y O’Reilly 1998). No obstante, en base al tipo de característica que estudiemos en relación a los miembros de un grupo, la diversidad afecta a los resultados de manera diferente. Así, tradicionalmente se hacen dos distinciones generales: 1) diversidad observable. Se refiere a la 
heterogeneidad entre miembros de un grupo en una categoría social que se fácilmente observable, tal como el género, edad, grupo étnico. Esta diversidad es relacionada negativamente con el desempeño del grupo ya que se ha demostrado que la gente tiende a favorecer más a los miembros de su grupo que a los de fuera de él, a confiar más y estar más dispuestos a cooperar con ellos (Brewer 1979, Brewer y Brown 1998, Tajfel y Turner 1986); y 2) diversidad no-observable, la cual se basa en atributos subyacentes a los individuos, tales como conocimiento, educación, valores, percepción, afecto y características personales. Esta diversidad está relacionada de manera positiva con el rendimiento de un grupo ya que se entiende que estos grupos son propensos a tener un rango más amplio de conocimientos relevante para ejecutar las tareas, mayor habilidades y capacidades, y miembros con diferentes opiniones (Boeker 1997; Watson, Johnson y Merritt 1998; Kilduff, Angelmar y Mehra 2000; Timmerman, 2000). El proyecto pretende evaluar el impacto en el rendimiento de los grupos de trabajo de ambos tipos de diversidad

Este objetivo general se concreta en los siguientes objetivos específicos:

1. Identificación de las variables sociales y funcionales que caracterizan a los alumnos mediante la recolección de datos primarios (a través de una encuesta inicial) y secundarios (a través de sus fichas de clase).

2. Crear un perfil social y funcional de cada alumno.

3. Determinar diferentes índices de diversidad para cada grupo en base una serie de variables funcionales y sociales.

4. Realizar correlaciones entre los diferentes índices y las notas obtenidas por cada uno de los grupos de trabajo en términos de evaluación media final.

5. Si las correlaciones fueran elevadas, planificar la organización de los grupos para los años posteriores.

\section{METODOLOGÍA Y ACTIVIDADES QUE SE VAN A DESARROLLAR}

Las diferentes actividades que se van a desarrollar son las siguientes:

\subsection{OBTENCIÓN DE INFORMACIÓN SOBRE EL PERFIL DE LOS DIFERENTES ALUMNOS.}

La información sobre cada alumno se va a obtener por medio de dos procedimientos. Por un lado, pasaremos una encuesta sobre algunos datos personales, que serán tratados según la ley de protección de datos, y por otro lado, recurriremos a la ficha electrónica y matrícula de cada uno de ellos. En concreto, debemos obtener información tanto de su perfil social como funcional. Del primero, pretendemos conseguir la edad, el género, la nacionalidad y el lugar de residencia, mientras que del segundo necesitamos los datos de su background educacional, a través del tipo de bachillerato estudiado, y 
funcional, si es la primera vez que cursa la asignatura.

\subsection{CÁlCULO DEL ÍNDICE DE DIVERSIDAD PARA CADA UNO DE LOS GRUPOS DE} TRABAJO.

Para calcular la diversidad, vamos a utilizar una versión del Herfindahl index (Daellenbach y McCarthy, 1999; Kor, 2006): $\mathrm{H}=1-\Sigma \mathrm{Pi}^{2}$, donde pi es la proporción de miembros en cada categoría. El valor de $\mathrm{H}$ varía entre 0 y 1 , valores cercanos a 1 indican alta diversidad.

Vamos a calcular diversos índices de diversidad agrupados en dos categorías: funcional y social

En la categoría social incluimos, diversidad de género, edad, nacionalidad y lugar de residencia. Pi es la proporción de miembros en cada categoría siendo para el género " $1=$ mujer" y " 0 =hombre"; para la edad " $1=18-20 "$, " $2=20-25$ " y " $3=$ otras edades", para la nacionalidad " $1=$ española y " $0=$ extranjera" y para el lugar de residencia "1=Sevilla", "2=Dos Hermanas", "3=otros".

En la categoría funcional incluimos el tipo de bachillerato estudiado y si es la primera vez que cursa la asignatura. Nuevamente, para el cálculo de la Pi, creamos las categorías las categorías de "1=bachillerato ciencias puras, "2=bachillerato letras" y " $3=$ bachillerato ciencias sociales" para la primera variable y las categorías de " $1=$ primera vez que cursa asignatura" y " $0=$ nunca ha cursado la asignatura" para la segunda de las variables a analizar.

\section{CÁlCulo de LAS CORRELACIONES ENTRE LOS DIFERENTES ÍNDICES DE DIVERSIDAD Y LAS NOTAS OBTENIDAS EN LAS CLASES PRÁCTICAS POR LOS ALUMNOS.}

Con ayuda del programa estadístico STATA 17.0 y a través de un análisis de correlación, pretendemos averiguar si diferentes índice de diversidad calculados para cada grupo de trabajo tienen influencia sobre sus resultados finales. Si es así, pretendemos reorganizar los grupos para el año siguiente de forma que se trate de optimizar su funcionamiento a partir de la composición de sus miembros. Como ya adelantamos, se pronostica que los dos tipos de diversidad analizadas van a tener influencias opuestas en los rendimientos del grupo por lo que las nuevas configuraciones podrán tener en cuenta este hecho y fomentar la diversidad funcional y minimizar la social.

\subsection{EVALUACIÓN.}

El proyecto pretende evaluar el impacto de la composición de los grupos de trabajo en su rendimiento. En concreto, se analizará la relación existente entre la diversidad funcional y social de cada 
grupo de trabajo y su rendimiento. En este sentido, se evaluará el rendimiento global del grupo de trabajo, medido como la nota media final obtenida en todas las EPDs del curso.

\section{RESULTADOS}

A raíz de la información recogida en los cuestionarios se crearon, en primer lugar, perfiles tanto funcionales como sociales de cada uno de los alumnos. Con carácter general, se puede decir que un $64 \%$ son mujeres y un $36 \%$ hombres, en su mayoría (58\%) con una edad comprendida entre 20 y 30 años. El 96\% vive en Sevilla o provincia siendo el mismo porcentaje de nacionalidad española.

Con respecto a los factores funcionales, podemos decir en referencia al tipo de bachiller que han cursado que un $16 \%$ provienen de ciencias puras, un $28 \%$ de letras, un $48 \%$ de ciencias sociales y un $8 \%$ de otra tipología. Un $72 \%$ es el primer grado que estudia frente a un $28 \%$ que previamente ha cursado otro grado universitario. En cuanto a experiencia laboral un $16 \%$ no posee ninguna experiencia, un $24 \%$ posee menos de un año y un $60 \%$ cuenta con más de un año de experiencia profesional. Con respecto a la relación con los compañeros, podemos decir que un $32 \%$ de ellos conoce previamente a sus compañeros (23\% solo a uno y $9 \%$ a más de uno) frente a un $68 \%$ que no conoce a ninguno.

En base a los dos tipos de diversidad que tratamos en nuestra propuesta, diversidad observable y diversidad no observable, se han construido diferentes índices de Blau. Así, por ejemplo, el género (0.46), la edad (0.49) y el lugar de residencia (0.57) tienen una diversidad media, frente a la poca diversidad que tiene la nacionalidad (0.08). En referencia a los factores funcionales, los estudios previos (0.40), la experiencia laboral (0.56) y la relación previa con los compañeros (0.44) presenta una diversidad media frente a los tipos de bachiller (0.66) que son un poco más diversos.

A través del programa estadístico Stata 17.0, se ha extraído una matriz de correlación entre las calificaciones de las prácticas (EPDs) y las diversidad social y funcional. Los resultados demuestran que en referencia a los factores sociales, la diversidad de género $(0.586 \mathrm{p}=0.02)$ tiene una relación positiva con las calificaciones grupales. Por el contrario la diversidad de nacionalidad $(-0.634 \mathrm{p}=0.001)$ tiene una relación negativa con el rendimiento del grupo. Factores como la diversidad de la edad $(0.313 \mathrm{p}=0.128)$ o la diversidad de lugar de residencia $(0.363 \mathrm{p}=0174)$ no tienen influencia en las notas del grupo. Con respecto a los factores funcionales, tanto la diversidad en los estudios previos $(-0.813 \mathrm{p}=0.000)$ como la diversidad en la experiencia laboral $(-0.442 \mathrm{p}=0.027)$ tienen una relación negativa con el rendimiento del grupo. No tienen influencia tampoco la diversidad en el tipo de bachiller $(0.147 \mathrm{p}=0.483)$ ni el conocer previamente a los compañeros $(0.001 \mathrm{p}=0.989)$. 


\section{CONCLUSIÓN}

Como conclusión, vemos que diferentes tipos de diversidad analizadas van a tener influencias diferentes en los rendimientos del grupo por lo que las nuevas configuraciones deben tener en cuenta este hecho. En concreto, creemos que los grupos de epd de años próximos deben ser organizados siguiendo los siguientes criterios:

Sociales:

- Grupos diversos en género

- Grupos con la misma nacionalidad

Funcionales:

- Estudios previos similares

- Experiencia laboral en el mismo nivel

El EEES utiliza las enseñanzas prácticas y desarrollo como pilar fundamental del aprendizaje autónomo. Estas actividades son realizadas, en gran medida, en grupo acercando al alumno la realidad de las organizaciones en las que probablemente ejerzan su futura actividad profesional. Por este motivo, conocer el impacto que la composición de los grupos de trabajo puedan tener tanto en el rendimiento final del grupo, como en la progresión en dicho rendimiento es fundamental para el éxito del aprendizaje asociado a cualquier asignatura, de cualquier grado y posgrado, que se apoye en la utilización de actividades prácticas y desarrollo en grupo como parte del proceso de transmisión de conocimientos y habilidades al alumno.

A todo ello, hay que añadir que esta experiencia no sólo permite acercarnos a las necesidades de cada individuo en particular, aspecto estudiado en numerosos proyectos de innovación, sino que nos va a permitir conocer la idiosincrasia de cada grupo de trabajo y adaptar las metodologías docentes a cada uno de ellos mediante el análisis de las características y elementos que los definen, un aspecto bastante descuidado y de máxima necesidad en el contexto actual. 


\section{REFERENCIAS}

Arroyo-Salgueira, S., Iglesias-Martin, M.J. and Lozano-Cabezas, I. (2021). os programas de movilidad estudiantil en la Educación Superior: el caso de una universidad española South Florida Journal of Development,.2, n.2, p.1474-1488.

Jackson, B.W., Lafasto, F. Schultz, H.G. and Kelly, D. (1992). Diversity, Human Resource Management, Volume31, Issue1-2, 21-34.

Triandis, H. C. (1994).Theoretical and methodological approaches to the study of collectivism and individualism. In U. Kim, H. C. Triandis, C. Ka@itçibaßi, S. C. Choi \& G. Yoon (eds), Individualism and Collectivism: Theory, Method and Applications, (pp. 41-51). Thousand Oaks, CA: Sage.

Williams, K. and O'Reilly, C.A. (1998). Demography and Diversity in Organizations: A Review of 40 Years of Research, Research in Organizational Behavior 20:77-140

Brewer, M. B. (1979). In-group bias in the minimal intergroup situation: A cognitive-motivational analysis. Psychological Bulletin, 86(2), 307-324. https://doi.org/10.1037/0033-2909.86.2.307

Brewer, M. B., \& Brown, R. J. (1998). Intergroup relations. In D. T. Gilbert, S. T. Fiske, \& G. Lindzey (Eds.), The handbook of social psychology (p. 554-594). McGraw-Hill

Tajfel, H. \& Turner, J. C. (1986). The social identity theory of inter-group behavior. In S. Worchel \& L. W. Austin (Eds.), Psychology of Intergroup Relations. Chigago: Nelson-Hall

Boeker, W. (1997). Strategic change: The influence of managerial characteristics and organizational growth. Academy of Management Journal, 40(1), 152-170. https://doi.org/10.2307/257024

Watson WE, Johnson L, Merritt D. Team Orientation, Self-Orientation, and Diversity in Task Groups: Their Connection to Team Performance Over Time. Group \& Organization Management. 1998;23(2):161-188. doi:10.1177/1059601198232005

Kilduff, M., Angelmar, R., \& Mehra, A. (2000). Top management-team diversity and firm performance: Examining the role of cognitions. Organization Science, 11(1), 21-34. https://doi.org/10.1287/orsc.11.1.21.12569

Timmerman TA. Racial Diversity, Age Diversity, Interdependence, and Team Performance. Small Group Research. 2000; 31(5):592-606. doi:10.1177/104649640003100505

Daellenbach, U., Mccarthy, A., \& Schoenecker, T. (1999). Commitment to Innovation: The Impact of Top Management Team Characteristics. $R \&$ D Management, 29, 199-208.

Kor, Y. Y. (2006). Direct and Interaction Effects of Top Management Team and Board Compositions on R\&D Investment Strategy. Strategic Management Journal, 27(11), 10811099. https://doi.org/10.1002/smj.554 